\title{
La protección a las víctimas de trata de personas en Brasil
}

\author{
Arisa Ribas Cardoso* \\ Danielle Annoni***
}

Recibido: mayo de 2015

Evaluado: agosto de 2015

Aprobado: noviembre de 2015

\section{RESUMEN}

La trata de personas es una acción ilegal presente en todo mundo, incluso en Brasil. El Protocolo de las Naciones Unidas contra la Trata de Personas fue ratificado por Brasil en 2004. A pesar de esto, aún la evolución normativa del país no ha sido significativa. Este artículo busca analizar la protección normativa existente y futura a las víctimas, especialmente extranjeras, de trata de personas en Brasil. Así, inicialmente se presenta lo que se comprende como trata de personas, con base en el Protocolo de las Naciones Unidas para prevenir, reprimir y sancionar la trata de personas, especialmente mujeres y niños, y son apuntadas algunas consideraciones sobre la realidad de la trata de personas en el mundo y en Brasil. En la secuencia son presentadas las medidas de protección a las víctimas, y previstas en el Protocolo de las Naciones Unidas. Por último, son enumeradas y analizadas las acciones normativas existentes y los proyectos de ley que pueden cambiar esta realidad. La conclusión del estudio indica que Brasil aún tiene una débil protección para las víctimas extranjeras de trata de personas, pero hay una perspectiva optimista de mejoría con los proyectos de ley que tramitan en el Congreso Nacional.

Palabras clave: Trata de personas; derecho internacional de los derechos humanos; Brasil.

El proyecto, El Estatuto de los Refugiados ¿puede ser aplicable a víctimas de trata de personas?, fue apoyado por CAPES y finalizado en 2014.

** Arisa Cardoso es mestre en Derecho y Relaciones Internacionales por la Universidad Federal de Santa Catarina. Es parte del grupo de investigacion Observatório de Derechos Humanos de UFSC. Contato: arisarc@ gmail.com.

*. Danielle Annoni é doctora en Derecho Internacional y professora permanente de Derecho Internacional y Derechos Humanos en la Universidad Federal del Paraná. Es titular de la Cátedra Sergio Vieira de Mello de ACNUR y Coordinadora del Observatório de Derechos Humanos de UFSC. Contato: danielle.annoni@gmail. com. 


\title{
Protection for Victims of Human Trafficking in Brazil
}

\begin{abstract}
Human trafficking is an illegal action observed in the whole world, even in Brazil. The Protocol of the United Nations against human trafficking was ratified by Brazil in 2004. However, the country has not shown a significant regulatory evolution. This article is intended to analyze present and future norms to protect victims (foreign victims, especially) of human trafficking in Brazil. First, the article presents what is understood as human trafficking, based on the protocol of the United Nations to prevent, repress, and penalize human trafficking (women and children, specifically); the article also describes some considerations about the reality of human trafficking in Brazil and worldwide. The sequence shows the measures taken to protect victims, contemplated in the United Nations protocol. Finally, the article provides a list of the existing regulatory actions and the bills than can change this reality. The conclusion of the study indicates that Brazil still offers a weak protection for foreign victims of human trafficking, but an optimistic improvement perspective is shown in relation to the bills currently analyzed by the National Congress.
\end{abstract}

Key words: Human trafficking; international human rights law; Brazil. 


\section{INTRODUCCIÓN}

La trata de personas es una modalidad de delincuencia transnacional que se fortaleció en la década de 1990 debido a una mayor facilidad de locomoción, así como a una disminución en la fiscalización en muchas regiones. En un primero momento, Brasil se tornó un país de origen de muchas víctimas de este tipo de criminalidad. Todavía, en el siglo XXI, con el desarrollo de la economía del país, pasó a ser también un destino de estas víctimas, especialmente oriundas de otros países de Sudamérica, como bolivianos, peruanos y paraguayos.

En razón de la gravedad de la trata de personas, esta modalidad de delincuencia fue elegida como una de las que tendría un Protocolo especial, anexo a la Convención de las Naciones Unidas contra la Delincuencia Organizada Transnacional ${ }^{1}$. Brasil ha ratificado el Protocolo contra la Trata de Personas en 2004. A pesar de esto, los esfuerzos para la implementación de una política pública sobre la temática solo han empezado en 2006. Sucede que esto ocurrió por medio de Decreto presidencial, medida que, aunque efectiva, no tiene la legitimidad democrática de

\footnotetext{
"The Organized Crime Convention is essentially an instrument of international cooperation, its stated purposes being to promote interstate cooperation in order to combat transnational organized crime more effectively. In this respect, its goal is to enlarge the number of States taking effective measures against transnational crime and to forge and strengthen cross-border links. More specifically, the Convention seeks to eliminate 'safe havens' where organized criminal activities or the concealment of evidence or profits can take place by promoting the adoption of basic minimum measures" (Gallagher, 2010, p. 74).
}

una ley y es fácilmente cambiable, lo que genera inseguridad jurídica y es bastante perjudicial para personas en situación de vulnerabilidad como las víctimas de trata. No obstante, se percibe un cambio en esta realidad, en razón de proyectos de ley que tramitan en el Congreso Nacional.

Este artículo, entonces, busca analizar la protección normativa existente y futura a las víctimas, especialmente extranjeras, de trata de personas en Brasil, bajo la perspectiva del Protocolo de las Naciones Unidas para prevenir, reprimir y sancionar la trata de personas, especialmente mujeres y niños. Así, inicialmente se presenta lo que se comprende como trata de personas, con base en el Protocolo, y se hacen algunas consideraciones sobre la realidad de la trata de personas en el mundo y en Brasil. En la secuencia son presentadas las medidas de protección a las víctimas, previstas en el Protocolo de las Naciones Unidas. Por último, son enumeradas y analizadas las acciones normativas existentes y los proyectos de ley que pueden cambiar la realidad.

\section{LA TRATA DE PERSONAS $Y$ LA TRATA DE PERSONAS EN BRASIL}

La trata de personas es una de las modalidades de delincuencia organizada que se han fortalecido con la globalización². Con el fin de la Guerra Fría el flujo de

\footnotetext{
2 "Human Smuggling and trafficking have been among the fastest growing forms of transnational crime because current world conditions have created increased demand and supply" (Shelley, 2010, p. 2).
} 
bienes y personas a través de las fronteras se ha acentuado significativamente, y la fiscalización ha disminuido. Por eso, ha sido elaborada la Convención de las Naciones Unidas contra la Delincuencia Organizada Transnacional y sus protocolos, incluso, el relacionado con la trata de personas.

El Protocolo de las Naciones Unidas para prevenir, reprimir y sancionar la trata de personas, especialmente mujeres y niños, define la trata como:

[...] la captación, el transporte, el traslado, la acogida o la recepción de personas, recurriendo a la amenaza o al uso de la fuerza u otras formas de coacción, al rapto, al fraude, al engaño, al abuso de poder o de una situación de vulnerabilidad o a la concesión o recepción de pagos o beneficios para obtener el consentimiento de una persona que tenga autoridad sobre otra, con fines de explotación. Esa explotación incluirá, como mínimo, la explotación de la prostitución ajena u otras formas de explotación sexual, los trabajos o servicios forzados, la esclavitud o las prácticas análogas a la esclavitud, la servidumbre o la extracción de órganos (UNODC, 2004, p. 44-45).

La trata de personas está, por lo tanto, compuesta por tres elementos: la acción, los medios y los propósitos (Gallagher, 2010; UNODC, 2012). La acción es la captación, el transporte, el traslado, la acogida o la recepción. Los medios son la amenaza o el uso de la fuerza u otras formas de coacción, el rapto, el fraude, el engaño, el abuso de autoridad o valerse de la situación de vulnerabilidad o de la concesión o recepción de pagos o beneficios para obtener el consentimiento de una persona que tenga autoridad sobre otra. El propósito es la explotación. Los tres elementos deben coexistir para que la conducta delincuente sea caracterizada, salvo en los casos que envuelven niños, cuando los medios, de acuerdo con la letra 'c' del artículo 3, del Protocolo, no son necesarios (Gallagher, 2010). Estos son los elementos que caracterizan la trata de personas y que, en consecuencia, determinan quiénes son sus víctimas.

La trata de personas ocurre en todo el mundo y varios patrones se renuevan. Existe gran dificultad en la identificación de este delito, así como en establecer estadísticas más precisas y estudios sobre el tema. La mayoría de los datos existentes provienen de la Unión Europea, donde la principal forma de trata de personas que ocurre es con fines de explotación sexual, lo que a veces puede enmascarar las estadísticas existentes (UNODC, 2012). Sin embargo, los casos de trata para trabajo forzado son cada vez más identificados, sobre todo en los principales países de destino, como Rusia o Estados Unidos, pero también, cada día con más frecuencia, en América Latina, incluso en Brasil. Según el informe sobre la Trata de Personas de la Oficina de las Naciones Unidas contra la Droga y el Delito [UNODC] (2012), la mayor parte de los flujos de trata son intrarregionales, o sea, las personas son llevadas para países dentro de su proprio continente. 
Las víctimas de trata de personas son también víctimas de una realidad social. En general se encuentran en una situación de vulnerabilidad ${ }^{3}$. Las debilidades se generan por cuestiones tanto económicas, como políticas, sociales o culturales. El subdesarrollo y la falta de perspectiva en algunos países y regiones hacen que la gente vaya a buscar oportunidades en otros lugares para mantener a sus familias. El trato desigual de los grupos étnicos o entre los géneros en algunos lugares también fomenta el surgimiento de poblaciones vulnerables. Las guerras civiles dejan familias sin sostén y crean demandas por soldados y mujeres.

Las personas mismas se ponen en situaciones de riesgo de trata. Cuando buscan facilitadores para viajar para otros países, por las más diversas razones -conseguir un trabajo mejor, o simplemente huir de una situación intolerable de miseria o explotación-, muchas veces terminan cayendo en estratagemas y en las manos de los tratantes que aprovechan las vulnerabilidades de esta gente para escoger sus víctimas y trazar sus estrategias de captación.

La trata no ocurre siempre de la misma manera. Existen diversos medios de cap-

3 "The rise of globalization and the widening of the gap between developing and developed countries, poverty, unemployment, and lack of equal opportunity are factors that contribute to the trafficking infrastructure. Innocent women and children become easy targets for traffickers, who take advantage of their economic vulnerability by offering illusory opportunities. Political instability, hostile occupation, armed conflict and civil unrest create social vulnerability of an insecure population that becomes disintegrated, displaced, and easily subjected to trafficking" (Mattar, 2008, p. 3). tación, traslado y explotación de las víctimas. Las principales víctimas son, en general, mujeres y niños, aunque el número de hombres sea también significativo. De acuerdo con UNODC (2012), en 2009, $59 \%$ de las víctimas eran mujeres; $17 \%$, niñas; $14 \%$, hombres, y $10 \%$, niños. De estas víctimas, en 2010, 58 \% eran tratadas para fines de explotación sexual; $36 \%$, para trabajos forzados; 0,2 \%, para remoción de órganos, y 6 \%, para otros fines. Las mujeres son tratadas especialmente, pero no exclusivamente, para prostitución, mientras los hombres son utilizados, en general, en trabajos forzados.

En las Américas las estadísticas del informe del UNODC (2012) no son muy diferentes de las globales. La mayoría de las víctimas son mujeres y niñas, y la principal forma de explotación es sexual (51 $\%)$; también, los casos relatados de trata para trabajos forzados son significativos (44 \%) y mayores que en otras regiones del mundo. Los flujos son principalmente intrarregionales, pero hay un número creciente de relatos de víctimas nativas de países del este asiático. En general, así como en los otros continentes, las personas migran de los países más pobres para aquellos que ostentan mejores condiciones económicas.

En los últimos años Brasil se ha convertido en un destino para las víctimas de trata internacional de personas debido a su desarrollo económico y mayor exposición en los medios de comunicación globales ${ }^{4}$.

\footnotetext{
$4 \quad$ "Brazil is a large source, destination, and transit country for men, women, and children subjected to sex trafficking and forced labor. A significant num-
} 
Las víctimas son generalmente de otros países del continente, como bolivianos o paraguayos, y más recientemente, los haitianos se han tornado, también, en presas fáciles para los tratantes de personas en Brasil.

Brasil es generalmente descrito como un país donde salen víctimas de trata internacional. Son frecuentes los relatos de mujeres brasileñas identificadas como víctimas de trata internacional en España u otros países de Europa, o aun en los Estados Unidos. En la América del Norte también fueron relatados casos de víctimas brasileñas de trata para trabajos forzados. La trata interna es del mismo modo un problema ya hace tiempo reconocido. En cambio, la preocupación con las víctimas extranjeras solo recientemente viene siendo debatida por los políticos y académicos.

En 2011, especialmente por la proximidad de la Copa del Mundo de Fútbol y de los Juegos Olímpicos, fue establecida

ber of Brazilian women and children are exploited in sex trafficking within the country, and federal police report higher rates of child prostitution in the Northeast. Brazilian women are found in sex trafficking abroad, often in Western Europe or neighboring countries such as Suriname, but also as far away as Japan. Authorities reported that between 2005 and 2011 the foreign ministry identified more than 300 Brazilian sex trafficking victims abroad. To a lesser extent, some women from neighboring countries, including Paraguay, have been exploited in sex trafficking in Brazil. Some transgender Brazilians are forced into prostitution within the country, and Brazilian men and transgender Brazilians have been exploited in sex trafficking in Spain and Italy. Child sex tourism remains a problem, particularly in resort and coastal areas in Brazil's northeast. Child sex tourists typically arrive from Europe and, to a lesser extent, the United States" (United Sates of America-USA-, 2013A, p. 103). una Investigación Parlamentaria ("Comissão Parlamentar de Inquérito" -CPI-) en el Senado "destinada a investigar la trata nacional e internacional de personas en Brasil, sus causas, consecuencias, rutas y responsables, en el período de 2003 y 2011, comprendido en la vigencia de la Convención de Palermo" (Senado, 2012, p. 1653. Traducción libre). En el informe final de esta investigación consta que el Oficial del Programa de la Unidad de Gobernanza y Justicia de la UNODC, Rodrigo Vitória, destacó que el país es una ruta tanto de origen como de destino para la trata de personas y que:

[...] las principales víctimas son mujeres y niños, y los fines son, fundamentalmente, explotación sexual y trabajo forzado. Afirmó que hay 110 (ciento y diez) rutas de trata interna y 131 (ciento treinta y una) de trata internacional, siendo que la región norte congrega la mayor cantidad de rutas. Aproximadamente el $80 \%$ (ochenta por ciento) de los casos son para fines de explotación sexual y las principales víctimas son mujeres entre 15 (quince) y 25 (veinte y cinco) años de edad. Ya sobre la trata para fines de trabajo forzado, la mayoría es de hombres y niños, principalmente de origen del Maranhão y del Piauí, con destino a Mato Grosso, Pará, área del Cerrado, Mata Atlántica y el Pantanal, haciendas, siendo que veinte cinco mil hombres estarían sujetos a esto trabajo forzado, especialmente en haciendas, campos de extracción de madera, cañaverales, y fincas productoras. En regla, la captación ocurre por medio de promesas de empleos falsos. Como destino, son involucradas mujeres, hombres y niños, personas 
de países vecinos, africanos y de China; y esencialmente para el trabajo forzado, Rio de Janeiro y São Paulo son los principales puntos. Informó, aún, que, especialmente después de la tragedia que sucedió en Haití, fueran identificados también casos de trata de niños haitianos para el Brasil, vía Guyana, para explotación sexual, especialmente en Amazonas y en los estados de São Paulo, Minas Gerais y Rio de Janeiro (Senado, 2012, p. 1676. Traducción libre.)

De acuerdo con otras contribuciones para la investigación, se destaca que las víctimas, en todas las modalidades de trata de personas, se encuentran en los grupos vulnerables de jóvenes, generalmente de bajos recursos, poca escolaridad y pocas oportunidades de mejoría de vida (Senado, 2012, p. 1677).

De acuerdo con el informe del Departamento de Estado de los Estados Unidos sobre la Trata de Personas, de 2013, Brasil es clasificado como Tier 2, que quiere decir: "Countries whose government do not fully comply with the TVPA's ${ }^{5}$ mínimum standards but are making significant efforts to bring themselves into compliance with those standards" (USA, 2013B, p. 44), porque:

The Government of Brazil does not fully comply with the minimum standards for the elimination of trafficking; however, it is making significant

TVPA (Victims of Trafficking and Violence Protection Act) es una ley americana que reglamenta el combate a la trata de personas en los Estados Unidos, y define acciones y patrones de combate para los otros países, muchos de ellos semejantes a los existentes en el Protocolo de las Naciones Unidas contra la trata de personas. efforts to do so. Authorities continued to prosecute and convict trafficking offenders, continued funding for 16 anti-trafficking offices, and increased awareness on trafficking in persons by launching well-publicized media campaigns about trafficking warning signs. The government also launched a national anti-trafficking plan and committed to spend the equivalent of approximately \$2.9 million to implement it by 2014 . Despite this progress, challenges remain. Brazilian officials continued to define trafficking as a movementbased crime and statutes prohibiting trafficking were both too broad and too narrow, making it difficult to assess fully government efforts to combat trafficking. The government continued to investigate and prosecute trabalho escravo cases, though sentences for some trabalho escravo offenders remained inadequate. The government also did not fund specialized services, such as shelters, for sex trafficking victim and did not report how many total sex trafficking victims were identified or referred to services during the year. Specialized services, including shelters and job training, were unavailable to many of the more than 2,600 potential labor trafficking victims identified during the year. Some identified foreign trabalho escravo victims were deported (USA, 2013A, p. 104).

O sea, Brasil tiene un número significativo de casos de trata de personas, tanto interna como internacional, y está implementando leyes y políticas públicas relacionadas con el tema, pero aún no posee mucha efectividad en las medidas desarrolladas. 


\section{LAS MEDIDAS PREVISTAS EN LA CONVENCIÓN $Y$ EN EL PROTOCOLO DE LAS NACIONES UNIDAS}

Brasil ratificó el Protocolo contra la Trata de Personas en 2004 y viene intentando implementar políticas públicas sobre el tema desde entonces, pero no logró cambios legislativos significativos hasta ahora. Es importante destacar que actualmente las discusiones sobre el tratamiento para los migrantes, refugiados y victimas de trata tiene gran relevancia en el ámbito nacional y están en curso modificaciones sustanciales en las leyes y reglamentos sobre estos temas ${ }^{6}$.

Como parte de la Convención de las Naciones Unidas contra la Delincuencia Organizada Transnacional y del Protocolo para prevenir, reprimir y sancionar la trata de personas, especialmente mujeres y niños ${ }^{7}$, Brasil debería implementar, por lo menos, algunas de las previsiones de estos tratados internacionales respecto a la protección de las víctimas de trata, ya que estas son víctimas de graves violaciones de derechos humanos y el país se presenta internacionalmente como fuer-

6 Entre los días 30/05//2014 y 01/06/2014 ocurrió la Conferencia Nacional sobre Migraciones y Refugio, en São Paulo, después de diversas etapas regionales, a fin de discutir y compilar las propuestas políticas relacionadas à cuestión migratoria en Brasil.

7 Un Estado solo pude ser parte de un de los protocolos de la Convención contra la Delincuencia Organizada Transnacional se fuera parte de esta, de acuerdo con el artículo 37 de la Convención. Aún, el Protocolo sobre Trata debe ser leído en conjunto con la Convención, se le aplicando las reglas generales de esta, siempre que posible. temente comprometido con el combate a las violaciones de derechos humanos.

En los artículos 24 y 25 de la Convención están previstas las disposiciones sobre la protección a los testigos y a las víctimas de la delincuencia organizada transnacional. En los casos de trata de personas, los principales testigos son las víctimas mismas y, de esta manera, las disposiciones referentes a estas también se les aplican. Cabe resaltar, no obstante, que más importante que la identificación como testigo, es la caracterización de ella como víctima, para fines de garantías más amplias de protección y tratamiento. Lamentablemente, esta no es práctica común en muchos países, que privilegian las personas que pueden ser más útiles para la investigación y persecución criminal, mientras que las más vulnerables son tratadas apenas como migrantes irregulares ${ }^{8}$.

El artículo 24 de la Convención aborda la cuestión de la protección de los testigos y, cuando fuere necesario, de sus familiares y personas próximas durante los procesos criminales referentes a las contravenciones previstas en ella, dentro de la capacidad de cada Estado. En el segundo párrafo del artículo, se ha observado que pueden ser incluidas medidas de protección física, en cuanto a la divulgación de la identidad y la localización de los

\footnotetext{
Las legislaciones de muchos países sobre la trata de personas traen privilegios para las víctimas que sirven como testigos en las investigaciones y pueden colaborar en la persecución criminal, mientras las víctimas que no quieren o no pueden ayudar son tratadas como un migrante más y, muchas veces, deportadas sin mayores cuidados.
} 
testigos, así como propiciar medios para la realización de las pruebas sin que ellos se expongan. Se sugiere, finalmente, que los Estados hagan acuerdos con otros Estados para facultar nuevo domicilio a estas personas, siendo que las disposiciones pueden ser aplicadas a las víctimas cuando fueren testigos.

El artículo 25, sobre la protección a las víctimas, fue redactado de la siguiente manera:

Artículo 25. Asistencia y protección a las víctimas

1. Cada Estado parte adoptará medidas apropiadas dentro de sus posibilidades para prestar asistencia y protección a las víctimas de los delitos comprendidos en la presente Convención, en particular en casos de amenaza de represalia o intimidación.

2. Cada Estado parte establecerá procedimientos adecuados que permitan a las víctimas de los delitos comprendidos en la presente Convención obtener indemnización y restitución.

3. Cada Estado parte permitirá, con sujeción a su derecho interno, que se presenten y examinen las opiniones y preocupaciones de las víctimas en las etapas apropiadas de las actuaciones penales contra los delincuentes sin que ello menoscabe los derechos de la defensa (UNODC, 2004).

O sea, cabe a los Estados elegir la forma que consideran más adecuada, dentro de sus posibilidades, para el tratamiento de las víctimas de trata encontradas en su territorio.

El Protocolo sobre la Trata de Personas no es muy diferente de la Convención en el tono, pero discrimina mejor ciertos derechos de las víctimas. El artículo 6, relativo a la asistencia y protección a las víctimas de la trata de personas establece:

Artículo 6. Asistencia y protección a las víctimas de la trata de personas

1. Cuando proceda y en la medida que lo permita su derecho interno, cada Estado parte protegerá la privacidad y la identidad de las víctimas de la trata de personas, en particular, entre otras cosas, previendo la confidencialidad de las actuaciones judiciales relativas a dicha trata.

2. Cada Estado parte velará por que su ordenamiento jurídico o administrativo interno prevea medidas con miras a proporcionar a las víctimas de la trata de personas, cuando proceda:

a) Información sobre procedimientos judiciales y administrativos pertinentes;

b) Asistencia encaminada a permitir que sus opiniones y preocupaciones se presenten y examinen en las etapas apropiadas de las actuaciones penales contra los delincuentes sin que ello menoscabe los derechos de la defensa.

3. Cada Estado parte considerará la posibilidad de aplicar medidas destinadas a prever la recuperación 
física, sicológica y social de las víctimas de la trata de personas, incluso, cuando proceda, en cooperación con organizaciones no gubernamentales, otras organizaciones pertinentes y demás sectores de la sociedad civil, y en particular mediante el suministro de:

a) Alojamiento adecuado;

b) Asesoramiento e información, en particular con respecto a sus derechos jurídicos, en un idioma que las víctimas de la trata de personas puedan comprender;

c) Asistencia médica, psicológica y material; $y$

d) Oportunidades de empleo, educación y capacitación.

4. Cada Estado parte tendrá en cuenta, al aplicar las disposiciones del presente artículo, la edad, el sexo y las necesidades especiales de los niños, incluidos el alojamiento, la educación y el cuidado adecuados.

5. Cada Estado parte se esforzará por prever la seguridad física de las víctimas de la trata de personas mientras se encuentren en su territorio.

6. Cada Estado parte velará por que su ordenamiento jurídico interno prevea medidas que brinden a las víctimas de la trata de personas la posibilidad de obtener indemnización por los daños sufridos (UNODC, 2004).

Las disposiciones, por lo tanto, se refieren a la protección de la privacidad y de la intimidad, la confidencialidad de los procedimientos judiciales, el derecho a la información sobre los procedimientos judiciales y administrativos aplicables, la asistencia para permitir la expresión de opiniones y preocupaciones durante el proceso criminal contra los autores del crimen, el auxilio para la recuperación física, psicológica y social, la posibilidad de indemnización por los daños sufridos; todo esto de acuerdo con el derecho interno del Estado y las posibilidades dentro de su sistema jurídico. Gallagher (2010) resalta que estas disposiciones son más flacas qua las de la Convención, en la cual está prevista la protección contra retaliación e intimidación, pues se refiere explícitamente a la protección de la integridad física.

La responsabilidad de proteger y de cuidar de las víctimas es de los Estados. Desde el momento en el que las autoridades saben de la existencia de víctimas de trata de personas, deben tomar todas las medidas posibles para preservar su seguridad e integridad. Además, no se puede ignorar que también después de rescatadas, las víctimas de trata continúan en situación de vulnerabilidad. Gallagher (2010, p. 297298) alerta que:

When considering the protection and support obligations owed to victims of trafficking it is important to acknowledge that their experience of harm does not necessarily cease when they come to the attention of national authorities. Corruption and complicity of public officials may result in a continuation of an exploitative situation or the emergence of a new one. The harm already done to victims can be compounded by failures to provide 
medical and other forms of support - or by linking the provision of such services to an obligation of cooperation that victims may not be willing or able to meet.

En este sentido son las principales críticas a las leyes de muchos países que condicionan el apoyo a las víctimas a su colaboración con los procedimientos de persecución. El artículo 7 del Protocolo sugiere a los Estados que consideren la posibilidad de conceder la permanencia temporaria o permanente a las víctimas de trata en su territorio, en los siguientes términos:

Artículo 7. Régimen aplicable a las víctimas de la trata de personas en el Estado receptor

1. Además de adoptar las medidas previstas en el artículo 6 del presente Protocolo, cada Estado Parte considerará la posibilidad de adoptar medidas legislativas u otras medidas apropiadas que permitan a las víctimas de la trata de personas permanecer en su territorio, temporal o permanentemente, cuando proceda.

2. Al aplicar la disposición contenida en el párrafo 1 del presente artículo, cada Estado Parte dará la debida consideración a factores humanitarios y personales (UNODC, 2004).

A través de este dispositivo se sugiere que los Estados adopten medidas que favorezcan a la víctima para permanecer en su territorio temporaria o permanentemente, así como que observen los factores humanitarios y personales. La observancia de esta disposición es fundamental para garantizar la dignidad de las víctimas de trata internacional de personas. Del mismo modo que el derecho a la identificación-que, a pesar de no estar explícito en el Protocolo, es una presuposición lógica de la aplicación de los demás derechos -, es este derecho que permitirá que los otros derechos sean disfrutados por la víctima. Una determinación de que esta persona pueda tener al menos un período de reflexión y recuperación es esencial; de otra manera el destino de la mayor parte de las víctimas, que por lo general no están regularmente en el país, es la deportación.

La permanencia temporaria en el país, con la debida asistencia, propicia que el Estado cumpla sus otras obligaciones. Así, la víctima podrá tener asistencia para su recuperación física y mental. La negación del permiso de permanencia, sea temporaria o definitiva, para las víctimas que pudieren y/o quisieren colaborar con las investigaciones o la persecución criminal, genera una desigualdad discriminatoria.

El artículo 8 del Protocolo trata de la repatriación, disponiendo sobre los deberes de los Estados cuando de este procedimiento se trata. Tanto el Estado del cual la víctima es nacional o tenga residencia permanente y para el cual está siendo repatriada, como aquel o donde ella se encuentra, deberán facilitar el proceso, incluso con la providencia de identificación y documentación, teniendo en cuenta la seguridad de ella. Es importante destacar que la repatriación debe 
ser hecha, preferencialmente, de manera voluntaria?.

Brasil, como un país comprometido con los derechos humanos y con recursos financieros razonables para políticas públicas sociales, debería y podría tener mejores leyes y una mejor estructura para combatir la trata de personas, dar tratamiento y acogida a sus víctimas, nacionales o extranjeras, pero, desafortunadamente, no es esta la realidad, aunque las perspectivas de cambio sean positivas.

\section{LA PROTECCIÓN EN BRASIL}

En términos de ley, Brasil posee dispositivos en el Código Penal sobre trata de personas, pero que no están de acuerdo con el Protocolo, aunque hayan sido cambiados después de la ratificación de este.

9 As noted previously, trafficked persons are routinely deported from countries of transit and destination. While States are able to point to a legal entitlement to control their own borders (and the absence of an obligation to permit all persons identified as having been trafficked to stay), there can be no doubt that forced repatriation to the country of origin or to a third country can have serious consequences for victims of trafficking. They may be subject to punishment from national authorities for unauthorized departure or other alleged offenses; they may face social isolation or stigmatization and be rejected by their families and communities; they may be subject to violence and intimidation from traffickers - particularly if they have cooperated with criminal justice agencies or owe money that cannot be repaid. Victims of trafficking who are forcibly repatriated, particularly without the benefit of supported reintegration, are at great risk of retrafficking. From a legal perspective, the issue of repatriation is a controversial one, involving consideration of complex issues such as entitlement to return and the principle of nonrefoulement" (Gallagher, 2010, p. 337).
Es oportuno destacar que a pesar de que la legislación penal no esté directamente relacionada con la protección a las víctimas, ella es esencial para la delimitación de la conducta delincuente considerada en cada país, ya que, aunque las políticas públicas presenten la definición del Protocolo, las autoridades tendrán más claridad para identificar las víctimas cuando exista un tipo penal adecuado.

El artículo 149, modificado en 2003, se refiere a la explotación de personas en situación análoga a la de esclavo:

Artículo 149. Reducir alguien a la condición análoga a la de esclavo, o sometiéndolo a trabajos forzados o a jornadas exhaustiva, o sometiéndolo a condiciones degradantes de trabajo, o restringiendo, por cualquier medio, su locomoción en razón de deuda contraída con el empleador o prepuesto:

Pena - reclusión, de dos a ocho años, y multa, además de la pena correspondiente a la violencia.

$\oint 1^{\circ}$ En las mismas penas incurre quien:

I - cercena el uso de cualquier medio de transporte por parte del trabajador, con el fin de retenerlo en el local de trabajo;

II - mantener vigilancia ostensiva en el local de trabajo o se apodera de documentos u objetos personales del trabajador, con el fin de retenerlo en el local de trabajo;

$\S 2^{\circ}$ La pena se aumentará en mitad si el crimen es cometido:

I - contra niños o adolescentes; 
II - por motivo de prejuicio de raza, color, etnia, religión u origen (Brasil, 1940. Traducción libre).

A pesar de que la modificación en el artículo mejoró su interpretación, no son considerados los procesos de transformación de las relaciones de trabajo en las últimas décadas, pues son criminalizados únicamente los agentes primarios sin llegar a los agentes secundarios o terciarios, en vista de los procesos de tercerización y subcontratación, que son estrategias de las corporaciones en la actualidad para disminuir sus costos y aumentar los lucros (Bignami, 2013). Así, en varias ocasiones, también los responsables por llevar las personas hasta el hogar donde serán explotadas, o sea, a veces agentes de la trata de personas, no serán punidos por la ley penal brasileña, ya que esta situación no está prevista en el artículo referente.

En el mes de junio de 2014 fue aprobado el proyecto de enmienda constitucional que modifica el artículo 243 de la Constitución brasileña para constar que las propiedades rurales y urbanas donde sea localizada explotación del trabajo esclavo serán expropiadas y destinadas a la reforma agraria y a programas de habitación popular, sin indemnización al propietario ${ }^{10}$. La aprobación de esta

10 El artículo 243 de la Constitución antes de la enmienda constitucional 81: "As glebas de qualquer região do País onde forem localizadas culturas ilegais de plantas psicotrópicas serão imediatamente expropriadas e especificamente destinadas ao assentamento de colonos, para o cultivo de produtos alimentícios e medicamentosos, sem qualquer indenização ao proprietário e sem prejuízo de outras sanções previstas em lei". Y después: "Art. 243. As propriedades rurais e urbanas de qualquer região do País onde forem localizadas culturas ilegais de enmienda es relevante en la lucha contra el trabajo esclavo, especialmente por los nuevos debates sobre el tema, pero apenas amplía el ámbito de la pena para los delincuentes, sin garantizar cualquier derecho para las víctimas.

Administrativamente, se ha logrado condenar a los explotadores a pagar los salarios y de la seguridad social a las víctimas como forma de reconstruir sus patrimonios. Las normativas del Ministerio del Trabajo son las reglas que orientan la actuación de los fiscales, quienes deberán responder por que estas víctimas, sean nacionales o extranjeras, sean tratadas de manera tal que se garanticen sus derechos, especialmente los relacionados con el derecho del trabajo (Bignami, 2013).

Los artículos 231 y 231-A del Código Penal Brasileño, modificado por la Ley 12.015 , de 2009, tipifica la trata internacional y la trata interna de personas para fines de explotación sexual, respectivamente:

Artículo 231. Promover o facilitar la
entrada, en el territorio nacional, de
alguien que viene para ejercer la pros-
titución u otras formas de explotación
sexual, o la salida de alguien que va a
ejercer en el extranjero.

Pena - reclusión de tres (3) a ocho (8) años.

plantas psicotrópicas ou a exploração de trabalho escravo na forma da lei serão expropriadas e destinadas à reforma agrária e a programas de habitação popular, sem qualquer indenização ao proprietário e sem prejuízo de outras sanções previstas em lei, observado, no que couber, o disposto no art. $5^{\circ}$. 
$\S 1^{\circ}$ Incide en la misma pena aquel que agencia, atrae o compra a la persona tratada, así como, teniendo conocimiento de esta condición, la trasporta, transfiere o acoge $[\ldots]$

Artículo 231-A. Promover o facilitar el dislocamiento de alguien dentro del territorio nacional para el ejercicio de la prostitución u otra forma de explotación sexual:

Pena - reclusión de 2(dos) a 6 (seis) años.

$\oint 1^{\circ}$ Incide en la misma pena aquel que agencia, atrae, vende o compra la persona tratada, así como, teniendo conocimiento de esta condición, trasportarla, transferirla o acogerla. [...] (Brasil, 1940. Traducción libre).

Hay agravantes, en los dos artículos: si la víctima es menor de 18 (dieciocho) años, si tiene problemas mentales, si hay relación de parentesco o deber de cuidado, protección o vigilancia, y si hay empleo de violencia, grave amenaza o fraude. Si el crimen es practicado para obtener ventaja económica, es también aplicada una multa.

Estos artículos no distinguen la prostitución voluntaria de la prostitución exploratoria, y no están de acuerdo con la definición de trata del protocolo que requiere los elementos de acción, medios y finalidad para la configuración de la conducta. De la forma como está escrita, la ley brasileña está más próxima a la conducta de tráfico de migrantes que a la de trata de personas, en los términos de la Convención de las Naciones Unidas y sus protocolos. Tampoco hay distinción respecto de la conducta de acoger, cuando es para ayudar y no para explotar.

Para la protección de las víctimas de estos delitos existen programas de asistencia, que son, en general, convenios del gobierno con ONG, especialmente para el acogimiento de niños y mujeres víctimas de explotación sexual. En este sentido, hay una razonable estructura para la defensa y recuperación de estas personas, pero no es suficiente.

Entre 1999 y 2003 funcionó el Programa Global de Prevención del Combate a la Trata de Seres Humanos (GPAT), del Ministerio de la Justicia. Este programa promovía diversas acciones, desde análisis de datos, la elaboración de informes, la capacitación de la sociedad civil, el acompañamiento de investigaciones y procesos, la promoción de campañas educacionales y preventivas, así como la revisión legislativa. Tuvo como resultado la formación de comités interinstitucionales y centros de referencia. Con el fin de dar continuidad al programa, los comités, en asociación con otras instituciones, continuaron los trabajos bajo la coordinación del Instituto Latino Americano de Promoción y Defensa de los Derechos Humanos -ILADH- (Ribeiro, 2010).

En 2006 fue lanzada la primera Política Nacional de Enfrentamiento a la Trata de Personas través del Decreto 5948 de 2006. La política tenía como finalidad establecer principios, directrices y acciones de prevención y represión a la trata de personas y de atención a las víctimas, conforme la 
legislación nacional y los tratados internacionales de derechos humanos, así como crear un grupo de trabajo para elaborar un Plan Nacional de Enfrentamiento a la Trata de Personas [PNETP].

Como directivas generales, entre otras, trazó: el fomento a la cooperación internacional; la articulación con ONG nacionales e internacionales; la estructuración de una red de enfrentamiento a la trata de personas; el fortalecimiento de la actuación en las regiones de frontera, puertos, aeropuertos, carreteras, estaciones de tren y de autobús, y otras áreas de interés; la verificación de la condición de la víctima y la respectiva protección y atención, en el exterior y en territorio nacional, así como su reinserción social; el incentivo a la realización de investigaciones; el incentivo a la formación y capacitación de profesionales para prevención y represión a la trata de personas, así como para la verificación de la condición de la víctima; la armonización de las legislaciones y procedimientos administrativos; el incentivo a la participación de la sociedad civil en las instancias de control social de las políticas públicas; el incentivo a la participación de órganos de clase y consejos profesionales en la discusión, y la garantía de acceso amplio y adecuado a informaciones en diferentes medios de comunicación (Brasil, 2006).

Entre las directivas específicas, conviene destacar las relacionadas con la atención a las víctimas, que están en el artículo 7 del Decreto y enumera las necesidades siguientes:
I- Protección y asistencia jurídica, social y de salud a las víctimas directas e indirectas de trata de personas;

II - Asistencia consular a las víctimas directas e indirectas de trata de personas, independientemente de su situación migratoria y ocupación;

III - Acogida y abrigo provisorio de las víctimas de trata de personas;

IV - Reinserción social con garantía de acceso a educación, cultura, formación profesional y para trabajo a las víctimas de trata de personas;

V - Reinserción familiar y comunitaria de niños y adolescentes víctimas de trata de personas;

VI - Atención a las necesidades específicas de las víctimas, con consideración especial en cuestiones de género, orientación sexual, origen étnico o social, procedencia, nacionalidad, raza, religión, edad, situación migratoria, actuación profesional u otros estatus;

VII - Protección de la intimidad y de la identidad de las víctimas de trata de personas; $\mathrm{y}$

VIII - Levantamiento, mapeo, actualización y divulgación de informaciones sobre instituciones gubernamentales y no-gubernamentales situadas en el Brasil y en exterior que prestan asistencia a las víctimas de trata de personas (Brasil, 2006).

De la lectura del Decreto se deprende que la política brasileña fue formulada conforme al Protocolo de las Naciones Unidas, 
y presenta numerosas directrices generales y específicas, así como propuesta acciones para los órganos y entidades públicos en el ámbito de sus respectivas competencias. El Decreto es bastante amplio, pero, concretamente solo previó la institución del Grupo de Trabajo para elaborar el PNETP.

El PNETP fue aprobado través del Decreto 6.347 y lanzado en 2008 para "reforzar y concretar los principios, directrices y acciones consagradas en la Política Nacional" (Brasil, 2008, p. V); tiene establecidas 100 (cien) metas, distribuidas en 11 (once) prioridades. En enero de 2010 fue presentado el Informe del Plan Nacional, donde se señaló la existencia de otros programas y proyectos de enfrentamiento a la trata de personas como el Programa Nacional de Seguridad Pública con Ciudadanía (PRONASCI) que es una política pública del Gobierno Federal de prevención y represión criminal que tiene el enfrentamiento a la trata de personas como una de sus acciones estructurales, así como los proyectos para modificar las legislaciones sobre extranjeros, el Disque Denúncia ${ }^{11}$, el Programa de Acciones Integradas y Referenciales de Enfrentamiento a la Violencia Sexual Infanto-Juvenil en el Territorio Brasileño (PAIR), el Plan Nacional de Políticas para las Mujeres, el Plan Nacional de Erradicación del Trabajo Esclavo (Brasil, 2010). El Informe también presentó las acciones concretas realizadas con base en el PNETP y su actual fase

" El Disque Denúncia es un número a que las personas pueden llamar para efectuar relatos de violaciones de Derechos Humanos, como violencia contra niños, mujeres. de ejecución. Se destacó en el Informe la creación de los Núcleos de Enfrentamiento a la Trata de Personas ${ }^{12}$ por medio de la Secretaría Nacional de Justicia/Ministerio de la Justicia en asociación con los gobiernos estaduales, una de cuyas funciones es planear las acciones de enfrentamiento al crimen en el ámbito local. Estos núcleos, con la Publicación de la Portaría 41/2009, pasaron a ser agentes articuladores y movilizadores de la Política Nacional (Brasil, 2010). Respecto a las víctimas, el informe destaca la actuación del Puesto Avanzado de Atención Humanizada a los Migrantes, que procura construir una metodología de atención para los migrantes brasileños deportados o inadmitidos y que retornan al país vía el Aeropuerto Internacional de Guarulhos, con énfasis en la atención a las mujeres y a los transexuales que presenten indicios de trata de personas (Brasil, 2010).

Después de la evaluación de la implementación del primer PNETP, en 2011, fue iniciada la construcción del segundo PNETP, lanzado en 2013. En este nuevo plan fueron destacados como objetivos: la ampliación y mejora de la actuación de los órganos relacionados; el fomento a la cooperación entre órganos públicos, sociedad civil y organizaciones internacionales; la reducción de las vulnerabilidades a la trata de personas; la capacitación de profesionales e instituciones; la producción y diseminación de informaciones; la sensibilización y movilización de la sociedad. Aun, el II PNETP apunta como líneas operativas: la mejora del marco re-

12 Hay hoy día dieciséis Núcleos de Enfrentamiento a la Trata de Personas en el país. 
gulatorio; la integración y fortalecimiento de las políticas públicas, redes de atendimiento y organizaciones; la capacitación para el enfrentamiento; la producción, gestión y diseminación de informaciones, y campañas y movilizaciones para el enfrentamiento (Brasil, 2013).

Este II PNETP está aún en implementación, pero los avances, especialmente respecto de la diseminación de informaciones sobre la trata de personas son notables. Las acciones concretas también han avanzado significativamente; no obstante, para las víctimas extranjeras, aún no existen tantos aparatos como para las nacionales, ya que Brasil es considerado y se ve más como un país de origen de víctimas que de destino, lo que no significa que no haya extranjeros que necesiten de apoyo especializado o de alternativas humanitarias para su permanencia en el territorio del país.

A pesar de no haber ley específica sobre la trata de personas en Brasil, existe una Resolución Normativa, 93 de 2010, del Consejo Nacional de Inmigración, órgano responsable de formular la política de inmigración vinculado al Ministerio del Trabajo, que prevé la concesión de visado para las víctimas de trata de personas por un año, período en que podrán decidir si colaborarán con las investigaciones (artículo 1). El pedido de visa, sin embargo, debe ser oriundo de las autoridades policiales o judiciales o del Ministerio Público (artículo 3), y treinta días antes de la expiración de este visado, la víctima deberá manifestar su voluntad de colaborar y, en caso positivo, será posible la prorrogación del permiso (artículo 4). Hay posibilidad, también, través de un concepto técnico dirigido al Ministerio de la Justicia por los órganos implicados en la atención a las víctimas de trata de personas (artículo 5), de la concesión del visado mismo a aquellas que no colaboraren con la investigación, cuando haya sido verificada la situación de vulnerabilidad social, económica o psicológica (artículo 6) (Ministério, 2010). Cuestiones burocráticas, como la necesidad de presentación de documento válido de viaje (artículo 7) pueden generar traspiés para la efectividad de la norma, ya que las víctimas de trata, muchas veces, tienen sus documentos confiscados o no los poseen.

En términos de perspectiva de cambios en la legislación nacional, se tramitan en el Congreso proyectos con disposiciones sobre la trata de personas. Uno que no traía muchos avances fue recientemente anexado a uno más elaborado, propuesto después por la CPI mencionada anteriormente. Hay otros dos, uno respecto a una nueva legislación para los extranjeros y otro sobre migraciones, que también presentan algunas disposiciones sobre la situación de las víctimas de trata.

La primera propuesta, de 2003, fue el Proyecto de Ley 2845 que objetivaba establecer normas para la organización y la manutención de políticas públicas específicas de prevención y enfrentamiento a la trata de personas, especialmente mujeres y niños, instituir el Sistema Nacional de Prevención y Enfrentamiento a la Trata de Seres Humanos y disponer sobre la reglamentación de sus aspectos civiles y 
penales (Câmara, 2003). Desafortunadamente, tal proyecto no presenta ninguna modificación sustancial en lo que ya existe en relación con las políticas públicas sobre la materia en el país; apenas confiere forma de ley para lo que ya está dispuesto en otros documentos, como los mencionados antes. Los pocos cambios de dispositivos penales sugeridos no reflejan la definición completa de trata de personas existente en el Protocolo de las Naciones Unidas. Es mantenida la cuestionable redacción de los dispositivos sobre la trata para explotación sexual. No hay mención alguna a la situación y tratamiento de las víctimas.

El mencionado Proyecto de Ley fue anexado al Proyecto de Ley 7.370/2014, resultado de la CPI del Senado sobre la Trata de Personas. En esto nuevo proyecto están previstos principios y directrices para el enfrentamiento a la trata de personas parecidos con los de la Política Nacional, pero hasta más amplios. Respecto a la atención a las víctimas, directas o indirectas, de la trata de personas, lo que dispone el proyecto es que esta comprenderá:

I - Asistencia jurídica, social y de salud;

II - Acogimiento en abrigo provisorio;

III - Atención a las necesidades específicas, especialmente en relación con cuestiones de género, orientación sexual, origen étnico o social, procedencia, nacionalidad, raza, religión, edad, situación migratoria, actuación profesional u otro estatus;

IV - Preservación de la intimidad y de la identidad; y
V - Prevención a la revictimización en la atención y en los procedimientos de investigación y judiciales.

$\S 1^{\circ}$ La atención a las víctimas se dará con la interrupción de la situación de explotación o violencia, la reinserción social, la garantía de facilitación de acceso a la educación, a la cultura, a la formación profesional y al trabajo y, en el caso de niños y adolescentes, a la busca de su reinserción familiar y comunitaria.

$\S 2^{\circ}$ En el exterior, la asistencia inmediata a las víctimas brasileñas estará a cargo de la red consular brasileña y será prestada independientemente de su situación migratoria, ocupación u otroestatus (Câmara, 2014. Traducción libre).

No hay diferencia significativa con relación a la política nacional, pero la aprobación por ley traería mayor seguridad para la continuación y aplicación de las directrices, una vez que la Política Nacional está basada en un decreto, lo cual es menos legítimo democráticamente que una ley y más fácilmente cambiable. Se percibe que el proyecto no expresa una preocupación especial para las víctimas extranjeras, las cuales, muchas veces, están en situación de mayor vulnerabilidad que las nacionales (cuando en Brasil), ya que, por veces, no poseen documentos, no hablan bien la lengua, no poseen familiares o amigos cerca. El proyecto apenas destaca la necesidad de atención específica y clama por la no discriminación, lo que es el mínimo esperado de un país comprometido con los derechos humanos. 
El proyecto presenta, aún, sugerencias de modificaciones en la legislación penal y procesal penal en consonancia con las previsiones de la Convención de las Naciones Unidas sobre la Delincuencia Organizada Transnacional, para permitir al juez determinar medidas relacionadas con los bienes de los investigados o acusados, cuando sean instrumento, producto o provecho del crimen, especialmente para pagar reparaciones de daños, prestaciones pecuniarias, multas y costas. Se prevé la inclusión de un nuevo crimen en el Código Penal, que sería el crimen de Trata de Personas, redactado, en el proyecto, de la siguiente manera:

\section{Trata de Personas}

Artículo. 149-A. Agenciar, atraer, reclutar, transportar, transferir, comprar, alojar o acoger persona, mediante grave amenaza, violencia, coacción, fraude o abuso, con la finalidad de:

I - removerle órganos, tejidos o partes de lo cuerpo;

II - someterla a trabajo en condiciones análogas a las de esclavo;

III - someterla a cualquier tipo de servidumbre;

IV - adopción ilegal; o

V-explotación sexual.

Pena - reclusión, de 4 (cuatro) a 8 (ocho) años, y multa.

$\S 1^{\circ}$ La pena es aumentada de un tercio hasta mitad si:

I- el crimen sea cometido por funcionario público en el desempeño de sus funciones o a pretexto de ejercerlas;
II - el crimen sea cometido contra niños, adolescentes o ancianos o con deficiencia;

III - el agente hace prevalecer relaciones de parentesco, doméstico, de cohabitación, de hospitalidad, de dependencia económica, de autoridad o de superioridad jerárquica inherente al ejercicio de empleo, cargo o función; o

IV - la víctima de trata de personas sea retirada del territorio nacional

$\S 2^{\circ}$ La pena es reducida de uno a dos tercios si el agente fuere primario y no integra organización criminosa (Câmara, 2014. Traducción libre).

Esta nueva tipificación sugerida abarca casi todos los elementos previstos en el Protocolo de las Naciones Unidas y aun incluye la adopción ilegal como uno de los fines de la trata que no están establecidos. Todavía, no hay la claridad de que cuando la víctima sea niño no son necesarios los medios para la caracterización del delito, lo que puede dificultar la condena en algunos casos, debido a los principios del derecho penal. Si tal propuesta fuere aprobada, habrá un gran avance en términos de identificación de situaciones de trata, así como mayores posibilidades de punición a los agentes de este delito.

Más importante que la tipificación, todavía, es la previsión de inclusión en el Estatuto del Extranjero de la posibilidad de concesión de residencia permanente a las víctimas de trata de personas en el territorio nacional, independientemente de su situación migratoria y de colaboración en procedimientos administrativos, 
policiales y judiciales. Aun, el proyecto incluye la posibilidad de concesión de visa para reunión familiar, sin la necesidad de pagar tasas o multas. El proyecto incluye también los trabajadores víctimas de trata en programas gubernamentales de seguro de desempleo (Câmara, 2014).

A su vez, en el Proyecto de Ley 5.655/2009, del nuevo Estatuto del Extranjero, hay disposición de que son extendidas a los extranjeros, independientemente de su situación migratoria, las medidas de protección a las víctimas y testigos de la trata de personas y del tráfico de migrantes. También está prevista la concesión, por el Ministerio de la Justicia, de residencia temporaria al extranjero víctima de trata de personas, independientemente de su situación migratoria. La residencia será, en regla, de un año. Cuando la víctima colabore con la investigación o con el proceso criminal, la residencia temporaria podrá ser prorrogada por un período más, en cuanto dure el hecho, y puede ser cambiada para residencia permanente. Hay previsión de garantía contra la deportación y repatriación, así como de que no habrá penalidades por infracciones administrativas prevista en la ley (Câmara, 2009).

Por último, el proyecto de ley del Senado, 288 de 2013, tiende a instituir la Ley de Migración y regular la entrada y estada de extranjeros en Brasil, y prevé medidas de protección a las víctimas y testigos de trata de personas y de migrantes. En este proyecto, las víctimas de trata de personas son elegibles para el visado permanente, mismo que sean migrantes irregulares en el país, debiendo tener en cuenta "la situación de vulnerabilidad social o económica o sicológica, entre otras, en su país de origen, que posibilite la revictimización, independientemente de colaborar con la investigación o proceso criminal", así como la existencia de coacción o grave amenaza, o la necesidad de asistencia en razón de la violencia sufrida. Para la identificación de la persona como víctima de trata es aplicable la definición del Protocolo de las Naciones Unidas (Senado, 2013). Estas son disposiciones bastante benéficas para las víctimas, pues la concesión de la permanencia en el país es independiente de su colaboración con la policía o las autoridades judiciales. En cuanto a las previsiones criminales, el proyecto no trae grandes avances; de hecho, tiene hasta algunas fallas terminológicas cuando denomina el crimen de trata de personas, lo que sería más correctamente, de acuerdo con los Protocolos de las Naciones Unidas, caracterizado como tráfico de migrantes. Se ve que la propuesta resultante de la CPI y la del Senado son más avanzadas en términos de protección a las víctimas de trata de personas en Brasil que la del nuevo Estatuto del Extranjero, una vez que prevén la posibilidad de concesión de permanencia a las víctimas sin necesitar que las mismas se dispongan a colaborar con las investigaciones. Tal hecho es realmente de elogio y será por cierto un ejemplo para otros países si se llegare a aprobar, pues la mayoría de ellos tiene (cuando tiene) disposiciones parecidas con las del proyecto del nuevo Estatuto del Extranjero, en lo cual solamente está prevista la concesión de visados para las 
víctimas que colaboraren con las investigaciones.

\section{CONSIDERACIONES FINALES}

Brasil se ha comprometido internacionalmente a combatir la trata de personas y a proteger a sus víctimas. Avances importantes fueran alcanzados en la Política Nacional, especialmente con la construcción de los Núcleos de Enfrentamiento, la ampliación de las campañas de concienciación, así como la especialización de profesionales para atender a las víctimas, como en los casos de atención humanizada en el Aeropuerto de Guarulhos, lo cual, desafortunadamente, es dirigido apenas a las víctimas brasileñas y no hay actitudes semejantes para los extranjeros.

Como se destacó, la preocupación con la situación de las víctimas extranjeras en territorio brasileño tuvo menos atención que otros aspectos, pero la existencia de la Resolución Normativa 93 del Consejo Nacional de Migración es una demostración de la preocupación del Estado brasileño con la situación de estas víctimas, especialmente cuando necesiten quedarse en el país. Todavía, debido a su naturaleza de reglamentación y no de ley, puede ser fácil y rápidamente cambiada, lo que genera inseguridad jurídica para esta población vulnerable.

Por fin, es importante notar que los proyectos de ley que están tramitando en el Congreso, como el resultante de la CPI y el del Senado, poseen una preocupación tanto con la redefinición del delito, para abarcar conductas antes no criminali- zadas, como con la protección de las víctimas, incluso cuando no quieren o no pueden colaborar con las investigaciones, lo que, con la aprobación de estas disposiciones, pondría a Brasil en el vanguardia en términos de protección humanitaria a estas víctimas, comparado con muchos otros países.

\section{REFERENCIAS}

Bignami, R. (2013). O Trabalho Escravo no Contexto do Tráfico de Pessoas: Valor do Trabalho, Dignidade Humana e Remédios Jurídico-Administrativos. In.: Secretaria Nacional de Justiça (Ed.). Tráfico de Pessoas: Uma abordagem para os Direitos Humanos. (pp. 475-506). Brasília, DF: Ministério da Justiça. http://monitoramentocedaw.com.br/wpcontent/uploads/2013/08/Cartilha_ TSH.pdf

Brasil. (1940). Decreto-Lei n. 2.848, de 7 de dezembro de 1940 [Versión Electrónica]. http://www. planalto.gov.br/ccivil_o3/decreto-lei/ del2848.htm

Brasil. (1988). Constituição da República Federativa do Brasil de 1988 [Versión Electrónica]. http:// www.planalto.gov.br/ccivil_ 03/constituicao/ constituicao.htm

Brasil. (2006). Decreto n. 5.948, de outubro de 2006 [Versión Electrónica]. http://www.planalto. gov.br/ccivil_03/_ato2004-2006/2006/ Decreto/D5948.htm.

Brasil. (2008). Plano Nacional de Enfrentamento ao Tráfico de Pessoas. [Versión Electrónica] http://www.unodc.org/documents/lpobrazil//Topics_TIP/Publicacoes/2008 PlanoNacionalTP.pdf

Brasil. (2010). Relatório Final de Execução do Plano Nacional de Enfrentamento ao Tráfico de Pessoas [Versión Electrónica] file:///C:/Users/Usuario/ Downloads/2010Relat\%C3\%B3rioPNET\%20 (2).pdf 
Brasil. (2013). II Plano Nacional de Enfrentamento ao Tráfico de Pessoas. [Versión Electrónica] file:///C:/Users/Usuario/Downloads/II\%20 Plano\%20Nacional.pdf

Câmara dos Deputados. (2003). Projeto de Lei $n^{\circ}$ 2.845/2003. [Versión Electrónica] http://www. camara.gov.br/proposicoesWeb/fichadetram itacao?idProposicao $=150103$

Câmara dos Deputados. (2009). Projeto de Lei $n^{0}$ 5.655/2009. [Versión Electrónica] http://www. camara.gov.br/proposicoesWeb/fichadetram itacao?idProposicao $=443102$

Câmara dos Deputados. (2014). Projeto de Lei $n^{0}$ 7.370/2014. [Versión Electrónical http://www. camara.gov.br/proposicoesWeb/fichadetram itacao?idProposicao $=611445 \&$ ord $=1$

Gallagher, A. T. (2010). The International Law of Human Trafficking [e-book]. New York: Cambridge University Press.

Mattar, M. Y. (2008). Trafficking in Persons: Global overview, current trends and pathways forward. [Versión Electrónica]. http:// www.protectionproject.org/wp-content/ uploads/2011/10/Trafficking-in-PersonsGlobal-Overview-Current-Trends-andPathways-Forward.pdf

Ministério do Trabalho e do Emprego. (2010). Resolução Normativa n. 93, de 21 de dezembro de 2010. [Versión Electrónica] http://portal.mte.gov.br/data/files/ FF8080812D5CA2D3012D60D125BF0640/ Resolu\%C3\%A7\%C3\%A30\%20Normativa\%20 n\%C2\%BA\%2093,\%20de\%2021_12_2010. pdf

Ribeiro, A. B. (2010). O Enfrentamento ao Tráfico de Pessoas no Brasil. In.: L. I. M. Júnior (Ed), Tráfico de Pessoas (pp. 67-96). São Paulo, SP: Quartier Latin.
Senado. (2012). Relatório Final: Comissão Parlamentar de Inquérito "destinada a investigar o tráfico nacional e internacional de pessoas no Brasil, suas causas, suas consequências, rotas e responsáveis, no período de 2003 e 2011, compreendido na vigência da Convenção de Palermo." [Versión Electrónica] http://www.senado.gov.br/atividade/materia/ getPDF.asp?t=121034Etp=1

Senado. (2013). Projeto de Lei do Senado n. 288, de 2013. [Versión Electrónical http://www. senado.gov.br/atividade/materia/getPDF. asp?t $=132518 \& \mathrm{tp}=1$

Shelley, L. (2010). Human Trafficking: A Global Perspective [e-book]. New York: Cambridge University Press.

United Nations Office for Drugs and Crime [UNODC]. (2004). Convención de las Naciones Unidas contra la Delincuencia Organizada Transnacional y sus protocolos [Versión Electrónica]. http://www.unodc.org/ documents/treaties/UNTOC/Publications/ TOC\%20Convention/TOCebook-s.pdf

United Nations Office for Drugs and Crime [UNODC]. (2012). Global report on trafficking in persons. [Versión Electrónica]. http://www. unodc.org/documents/data-and-analysis/ glotip/Trafficking _ in _ Persons _ 2012 _ web.pdf

United States of America -USA-. (2013). Country Narratives. [Versión Electrónica] http://www. state.gov/documents/organization/210738. $\underline{\mathrm{pdf}}$

United States of America -USA-. (2013). Trafficking in Persons Report. [Versión Electrónica] http://www.state.gov/documents/ organization/210737.pdf 